\title{
Fachgruppe Entwicklungspsychologie
}

\section{Kommentar zu Lindner, M. A. et al. (2021). Ein Plädoyer zur Qualitätssicherung schriftlicher Prüfungen im Psychologiestudium}

\author{
Julian Busch¹, Franziska Degé2, Julia Karbach ${ }^{3}$, Gisa Aschersleben ${ }^{4}$ und Mirjam Ebersbach ${ }^{5}$ \\ ${ }^{1}$ Ruhr-Universität Bochum \\ ${ }^{2}$ Max-Planck-Institut für empirische Ästhetik, Frankfurt \\ ${ }^{3}$ Universität Koblenz-Landau \\ «Universität des Saarlandes, Saarbrücken \\ ${ }^{5}$ Universität Kassel
}

Der Beitrag von Lindner et al. (2021) greift aus Sicht der Fachgruppe Entwicklungspsychologie mit der Qualitätssicherung bei schriftlichen Prüfungen einen wichtigen Aspekt des Psychologiestudiums auf. Die Autor_innen liefern eine Vielzahl praktischer Empfehlungen, um die Qualität solcher Prüfungen sicherzustellen bzw. zu erhöhen. Wir möchten in unserem Kommentar den Fokus der Diskussion um zwei wesentliche Aspekte erweitern: das
Problem der Noteninflation und eine bislang fehlende systematische Gegenüberstellung der Vor- und Nachteile unterschiedlicher Prüfungsformate.

Bei der Leistungsbeurteilung von Psychologiestudierenden ist die deutliche Tendenz zu beobachten, dass nur gute und sehr gute Noten vergeben werden (Noteninflation). So hat Antoni (2019) anhand einer Übersicht der aktuellen Studienabschlussnoten von Psychologiestu- 
dierenden auf eine mangelnde Differenzierung zwischen Absolvent_innen bezüglich ihrer Leistungsbeurteilung hingewiesen. Dabei zeigte sich auch, dass Universitäten in den vergangenen Jahren im Vergleich zu den (Fach-) Hochschulen häufiger Bestnoten vergeben haben (38\% vs. $27 \%$ ) und seltener die Abschlussnote "befriedigend" (5\% vs. $10 \%)$. Abschlüsse mit der Note "ausreichend“ wurden an beiden Institutionen überhaupt nicht vergeben. Darüber hinaus scheint fachübergreifend die Tendenz zur Noteninflation in Masterstudiengängen stärker zu sein als in Bachelorstudiengängen (IWD, 2013). Entsprechend sind Noten nicht normalverteilt, sondern stark rechtsschief. Interessanterweise ist das Problem der Noteninflation in der Psychologie deutlich größer als in anderen Fächern. So war 2012 der Anteil der Studierenden in der Psychologie, die ihren Abschluss mit Bestnote machten (48\%), deutlich höher als der Anteil der Studierenden in der Humanmedizin (7\%; IWD, 2013). Auf der Seite der Lehrenden spiegelt die Noteninflation strukturelle Herausforderungen in der psychologischen Lehre wider. Auf der Seite der Studierenden erhöht die Noteninflation den Druck auf Studierende, schränkt ihre intrinsische Studienmotivation sowie ihre Initiative zur fachlichen und methodischen Profilbildung ein. Dieser Tendenz kann nur begrenzt durch die Qualitätssicherung schriftlicher Prüfungen begegnet werden.

Neben der kritischen Betrachtung schriftlicher Prüfungsformate (Lindner et al., 2021) sollten daher auch weitere Mechanismen beachtet werden, die zu verzerrten Mustern von Leistungsbeurteilungen beitragen können. Ein solcher Mechanismus ist die Bezugsnorm. Werden die Leistungen der Psychologiestudent_innen mit einer hypothetischen Population aller Personen verglichen, die sich für das Studium beworben haben (soziale Bezugsnorm), ist eine solche Notenverteilung möglicherweise gerechtfertigt, da der Numerus Clausus für eine Bestenauslese sorgt. Allerdings erklärt dieser Ansatz nicht, dass in der Psychologie deutlich mehr Bestnoten vergeben werden als in der Humanmedizin. Legt man hingegen eine sachliche Bezugsnorm bei der Leistungsbeurteilung von Psychologiestudierenden zugrunde, d.h. vorab festgelegte Fähigkeiten und Kompetenzen, sollte eine solche Verteilung anders aussehen - besonders, wenn zwischen Studierendenleistungen differenziert und gleichzeitig ein hoher Qualitätsstandard gehalten werden soll. Für eine derartige Differenzierung spricht neben der verbesserten Beurteilungsfairness für Studierende auch ein höherer diagnostischer Wert im Hinblick auf künftige Leistungen in Studium und Beruf.
Der Gedanke einer sachlichen Bezugsnorm scheint jedoch aktuell im deutschen System teilweise konterkariert zu werden. Darauf deuten Unterschiede in den Leistungsbeurteilungen zwischen Standorten hin. Diese sind unter anderem darauf zurückzuführen, dass an manchen Standorten relative Bestehensgrenzen vorgesehen sind, die sich an der mittleren Leistung einer Kohorte orientieren und damit die soziale Bezugsnorm zugrunde legen. Dies bedeutet konkret, dass weniger als 50\% der Punkte in einer Klausur zum Bestehen ausreichen, wenn die Klausur insgesamt schlecht ausfällt. Dass das nicht nur an einer zu schweren Klausur, sondern auch an einer mangelnden Prüfungsvorbereitung oder -motivation liegen kann, wird bei der relativen Bestehensgrenze nicht berücksichtigt. Wir plädieren daher für ein Ausnutzen der gesamten Notenskala in Prüfungen, um die 3:06 unterschiedlichen Leistungen von Psychologiestudierenden trennschärfer und stärker kriteriumsorientiert abbilden zu können. Auf diese Weise könnte der Leistungsdruck und damit lediglich external motiviertes, auf Noten ausgerichtetes Lernverhalten von Studierenden reduziert werden, indem z.B. nicht mehr der Unterschied einer Zehntelnote über einen Masterstudienplatz entscheidet. Als Argument für die Verteilung guter Noten wird häufig auch angeführt, dass die "guten“ Bachelorstudierenden am eigenen Standort für den Masterstudiengang gehalten werden sollen. Um dies trotz Ausnutzung der Notenskala zu erreichen, bedarf es alternativer Auswahlkriterien für den Masterstudiengang. Denkbar wären hier z.B. an sachlichen Kriterien orientierte Auswahlklausuren (s. z. B. Uni Heidelberg) oder auch weitere standortspezifische Qualifikationen, die in die Auswahl einbezogen werden.

Qualitativ hochwertige Leistungsbeurteilungen erfordern, wie im Beitrag von Lindner et al. (2021) ausgeführt, auch ein hohes zeitliches und fachliches Engagement. Der wissenschaftliche Nachwuchs, der maßgeblich in die Durchführung und Beurteilung von Prüfungsleistungen eingebunden ist, hat jedoch zunehmend Schwierigkeiten, diesen hohen Anforderungen gerecht zu werden. Erstens hat die Anzahl der Prüfungen durch den Bologna-Prozess deutlich zugenommen. Grundsätzlich ist dies im Sinne eines (über die Studienzeit gesehenen) formativen Assessments zu begrüßen. Parallel dazu haben sich in den letzten 15 Jahren aber auch die Studierendenzahlen im Fach Psychologie vervielfacht und damit einhergehend die Studierenden-Professor_innen-Relation ungefähr verdoppelt $^{1}$ (Antoni, 2019; Wissenschaftsrat, 2018). Aus beiden Entwicklungen resultiert eine Verdichtung von Lehre und Leistungsbeurteilung.

Dies ist zwar größtenteils auf private Hochschulen zurück zu führen, jedoch steigen die Studierendenzahlen an den staatlichen Hochschulen ebenfalls an. 
Zweitens ist der wissenschaftliche Nachwuchs kontinuierlich gefordert, das zunehmend verdichtete Arbeitspensum von Lehrtätigkeit und studentischer Leistungsbeurteilung in Einklang mit den eigenen Qualifikationszielen zu bringen, da die Anstellung im Mittelbau im deutschen System ganz überwiegend ein berufliches Durchgangsstadium ist und der Verbleib in der Wissenschaft maßgeblich von der zügigen Eigenqualifikation abhängt (nur 1.1 bis $7.8 \%$ der Wissenschaftler_innen im Mittelbau sind aktuell entfristet tätig; Rentzsch, Harzer \& Wolker, 2017). Dabei legen formelle und informelle Anreizsysteme nahe, begrenzte Ressourcen stärker auf Forschungsaktivitäten als auf Lehre und studentische Leistungsbeurteilung $\mathrm{zu}$ verteilen. So sind die Qualität und Quantität von Publikationen sowie eingeworbene Drittmittel die zentralen Kriterien bei der Berufung auf eine Professur. Gerade an Standorten und in Studiengängen mit besonders vielen Studierenden kann jedoch die Arbeitsverdichtung für den wissenschaftlichen Nachwuchs besonders hoch und daher die Notwendigkeit, Kompromisse zwischen dem Aufwand für Forschung und Lehre zu finden, besonders bedeutsam sein.

Drittens werden studentische Lehrevaluationen als Kriterium in Berufungsverfahren herangezogen (für eine kritische Betrachtung der Aussagekraft von Lehrevaluationen, s. Feistauer \& Richter, 2017). Die Sorge von Nachwuchswissenschaftler_innen, dass sie negative Lehrevaluationen erhalten könnten, hält sie vermutlich auch davon ab, das gesamte Notenspektrum auszunutzen. Andernfalls könnten Sie gegenüber Mitbewerber_innen in Berufungsverfahren einen Nachteil haben.

Insgesamt können die beschriebenen Rahmenbedingungen, in denen Lehre und Leistungsbeurteilung aktuell stattfinden, zur Bedrohung von Qualitätsstandards in Lehre und studentischer Leistungsbeurteilung beitragen. Wir möchten daher anregen, ergänzend die Rolle des wissenschaftlichen Nachwuchses sowie Beziehungen zwischen Anstellungsverhältnissen und studentischer Leistungsbeurteilung in den Blick zu nehmen. Dazu wurden kürzlich auch Reformen angeregt (s. Positionspapier; Deutscher Hochschulverband, 2020, Abs. 5bb). Einerseits wird vorgeschlagen, dass Angestellte im Mittelbau mit erhöhtem Lehrdeputat häufiger entfristet werden, um dann unabhängiger in Lehre und studentischer Leistungsbeurteilung tätig zu sein. Andererseits wird vorgeschlagen, die wissenschaftliche Eigenqualifikation stärker von der wissenschaftlichen Dienstleistung zu entkoppeln, zu der Lehre und studentische Leistungsbeurteilung zählen, indem unterschiedliche Stellenformate geschaffen werden. Falls dann ein Großteil von Lehre und studentischer Leistungsbeurteilung von Personen ausgeübt würde, die vorwiegend $\mathrm{zu}$ diesem Zweck an Hochschulen angestellt sind, wäre denkbar, dass Lehrevaluationen kaum noch
Bedeutung für Berufungsverfahren für Professuren haben.

Neben der Diskussion von strukturellen Ursachen der Noteninflation, welche auf schriftliche wie mündliche Prüfungsformate zutrifft, möchten wir zuletzt den Blick auf unterschiedliche Prüfungsformate richten. Ein erheblicher Anteil von Prüfungsleistungen fußt auf Studierendenreferaten und Single- / Multiple-Choice Klausuren und seltener auf Hausarbeiten oder mündlichen Prüfungen. Ein Grund dafür könnte sein, dass Referate, die durch Studierende gehalten werden, die Lehrtätigkeit ökonomisieren, da sie zur Sitzungsgestaltung beitragen und von Lehrenden in-situ beurteilt werden können. Alternative Prüfungsformate (z.B. Portfolios, Anwendungsaufgaben, schriftliche Hausarbeiten) hingegen erfordern einen deutlich höheren Aufwand zur Beurteilung und werden somit seltener eingesetzt. An dieser Stelle wäre aus unserer Sicht eine systematische Gegenüberstellung der Vor- und Nachteile unterschiedlicher schriftlicher und mündlicher Prüfungsformate durch Expert_innen wünschenswert. Diese Gegenüberstellung sollte ergänzend feststellen, inwiefern schriftliche versus mündliche Prüfungen im Studium den tatsächlichen Lernerfolg im Sinne von Wissen, Verständnis und Transfer abbilden können. Nur so kann angemessen entschieden werden, welche Prüfungsformate einen gegebenenfalls höheren Aufwand seitens der Lehrenden rechtfertigen und an welcher Stelle sie sogar geboten sind. Die Definition von sachlichen Bezugsnormen für unterschiedliche Kompetenzbereiche könnte hier ein Ansatz zur Qualitätssicherung darstellen.

Zusammenfassend lässt sich festhalten, dass die Leistungsbeurteilung Studierender nicht nur in der Expertise unseres Fachs liegt, sie ist auch von zentraler Bedeutung für die Zukunft unseres Fachs. Wir möchten daher auf Basis des Artikels von Lindner et al. (2021) eine grundlegende Diskussion darüber anstoßen, wie Qualitätsstandards bezüglich der studentischen Leistungsbeurteilung generell gesichert werden können. Dies erfordert aus unserer Sicht (1) strukturelle Ursachen für aktuelle Tendenzen in verzerrten Leistungsbeurteilung von Psychologiestudierenden zu eruieren (Noteninflation, Einschränkung von Prüfungsformaten) und diesen entgegenzusteuern, (2) die wichtigen Darstellungen zur Qualität schriftlicher Prüfungen (Lindner et al., 2021) auf alternative Prüfungsformate auszuweiten und miteinander in Beziehung zu setzen, und (3) dazu anregen, Gestaltungspielräume in der Prüfungsgestaltung zu nutzen, um der Bedrohung qualitativ hochwertiger Leistungsbeurteilungen mit validen und reliablen Formaten proaktiv zu begegnen. 


\section{Literatur}

Antoni, C. H. (2019). Zur Lage der Psychologie. Psychologische Rundschau, 70, 4-26. https://doi.org/10.1026/0033-3042/ a000429

Deutscher Hochschulverband (2020, Februar). Resolution zum Befristungsrecht in der Wissenschaft - Positionspapier des Deutschen Hochschulverbands. Verfügbar unter: https://www. hochschulverband.de/positionen/presse/resolutionen/zum-be fristungsrecht-in-der-wissenschaft

Feistauer, D. \& Richter, T. (2017). How reliable are students' evaluations of teaching quality? A variance components approach. Assessment \& Evaluation in Higher Education, 42, 1263-1279, https://doi.org/10.1080/02602938.2016.1261083

Lindner, M. A., Sparfeldt, J. R., Köller, O., Lukas, J. \& Leutner, D. (2021). Ein Plädoyer zur Qualitätssicherung schriftlicher Prüfungen im Psychologiestudium. Psychologische Rundschau, 72, 93 - 105. https://doi.org/10.1026/0033-3042/a000524
Informationsdienst Wissenschaft (IWD) (2013, Oktober). Noteninflation. Verfügbar unter: https://www.iwd.de/artikel/noteninfla tion-128025/

Rentzsch, K., Harzer, C. \& Wolker, I. (2017). Stellungnahme zur Lage des wissenschaftlichen Nachwuchses in der Psychologie. Psychologische Rundschau, 68, 251-263. https://doi.org/10. 1026/0033-3042/a000377

Wissenschaftsrat (2018, Januar). Perspektiven der Psychologie in Deutschland. Verfügbar unter: https://www.wissenschaftsrat. de/download/archiv/6825-18.pdf?__blob=publicationFile\&v=1

\section{Dr. Julian Busch}

Arbeitsgruppe Child and Family Research

Ruhr-Universität Bochum

Universitätsstraße 150

44801 Bochum

Julian.Busch@rub.de

https://doi.org/10.1026/0033-3042/a000527 